\title{
Effects of metformin and thiazolidinediones on suppression of hepatic glucose production and stimulation of glucose uptake in type 2 diabetes: a systematic review
}

Received: 23 June 2005 / Accepted: 12 September 2005 / Published online: 14 February 2006

C) Springer-Verlag 2006

\begin{abstract}
Aims/hypothesis: Insulin resistance, which manifests itself as endogenous glucose overproduction and reduced insulin-mediated glucose uptake, is a core defect in type 2 diabetes. Metformin and the peroxisome proliferator-activated receptor- $\gamma$ agonists, the thiazolidinediones (TZDs), both lower glucose, although their mechanism of action is still subject to debate. This review analyses the evidence relevant to these mechanisms in vivo. Materials and methods: A systematic search of MEDLINE identified a total of 42 clinical studies that investigated the effects of TZDs $(n=23)$ and/or metformin $(n=19)$ on endogenous glucose production (using tracer glucose techniques) and peripheral glucose disposal (using the euglycaemic-hyperinsulinaemic clamp) in patients with type 2 diabetes $(n=549)$. The original variables assessed were converted into standardised units and their mean group values were listed separately for open and placebo-controlled studies. Statistical analysis was carried out, treating mean group values as individual values and comparing results (both as absolute values and percentage changes from baseline) across study categories (open vs placebo-controlled, TZDs vs metformin). Results: Both TZDs and metformin enhance insulin suppression of endogenous glucose production and fasting plasma glucose clearance. TZDs, but not metformin, also improve
\end{abstract}

Electronic Supplementary Material Supplementary material is available for this article at http://dx.doi.org/10.1007/s00125-0060141-7

\footnotetext{
A. Natali $\cdot$ E. Ferrannini $(\bowtie)$

Department of Internal Medicine,

University of Pisa School of Medicine,

Via Roma 67,

56126 Pisa, Italy

e-mail: ferranni@ifc.cnr.it

Tel.: +39-050-553272

Fax: +39-050-553235

E. Ferrannini

CNR Institute of Clinical Physiology,

Pisa, Italy
}

insulin-mediated glucose uptake at all insulin levels. Conclusions/interpretation: In patients with type 2 diabetes, metformin improves fasting hepatic insulin sensitivity and glucose clearance; TZDs improve fasting hepatic insulin sensitivity and glucose clearance, and potentiate glucose disposal under insulinised conditions.

Keywords Insulin resistance Metformin ·

Thiazolidinediones $\cdot$ Peroxisome proliferator-activated receptor- $\gamma$ agonists $\cdot$ PPAR $\gamma$

Abbreviations DB: double-blind - EGP: endogenous glucose production $\cdot$ ESM: electronic supplementary material $\cdot$ FFM: fat-free mass $\cdot M\left(R_{\mathrm{d}}\right)$ : insulin-stimulated glucose utilisation ( $M$ value or $R_{\mathrm{d}}$ ) PC: placebocontrolled - PI: production index - PPAR: peroxisome proliferator-activated receptor -TZD: thiazolidinedione

\section{Introduction}

Insulin resistance is a core defect in type 2 diabetes [1-3]. It contributes to hepatic glucose overproduction and is the major cause of decreased insulin-mediated glucose uptake by peripheral tissues. Pancreatic beta cells are able to maintain normoglycaemia in the face of insulin resistance by increasing insulin secretion. However, this compensatory response - for genetic or acquired reasons - might either be inherently limited or decline with time, eventually leading to hyperglycaemia.

A number of agents are available for the treatment of diabetes. Among them, the biguanide metformin is widely regarded as the standard first-line agent. This drug was first licensed as an antihyperglycaemic medication in Europe more than 40 years ago, at a time when its mechanism of action was little known. Since then, despite limited evidence, it has become recognised that metformin suppresses hepatic glucose production [4-6]. Claims that it improves peripheral insulin sensitivity have been made $[7,8]$, but remain controversial $[9,10]$, as does its effect on glucose absorption $[11,12]$. 
Peroxisome proliferator-activated receptor- $\gamma$ (PPAR $\gamma$ ) agonists were introduced more recently. This class of agents is currently represented by the thiazolidinediones (TZDs). The insulin-sensitising effect of TZDs was shown in preclinical studies, but detailed knowledge regarding the hepatic and peripheral insulin-sensitising activity of TZDs in vivo in man was lacking when these agents first entered clinical use.

To our knowledge, a systematic review of the clinical studies that have evaluated the effect of PPAR $\gamma$ agonists and metformin on hepatic and peripheral insulin sensitivity has not previously been conducted. Among clinicians, the prevailing paradigm appears to be that TZDs act on peripheral tissues (mainly adipose tissue), while metformin acts on the liver [6]. In this review we critically assess the validity of this paradigm by analysing published data from a total of 42 clinical studies that, by using the hyperinsulinaemic clamp and/or isotope dilution techniques, have investigated the effects of TZDs $(n=23)$ or metformin $(n=19)$ on hepatic and peripheral glucose metabolism.

\section{Materials and methods}

Identification of relevant publications We conducted a systematic search of MEDLINE (up to December 2004) to identify studies that investigated the mode of action of PPAR $\gamma$ agonists (TZDs) and metformin in patients with type 2 diabetes. Key search terms were 'metformin', 'thiazolidinedione', 'troglitazone', 'pioglitazone' or 'rosiglitazone' plus 'insulin resistance' or 'insulin sensitivity' or 'insulin action' or 'glucose production'. This search was restricted to English language publications involving patients with type 2 diabetes who had undergone at least one of two investigations: (1) measurement of insulin sensitivity by the hyperinsulinaemic glucose clamp technique; and (2) measurement of endogenous glucose production (EGP) using the tracer glucose technique. The search was completed by hand check on the references of these publications and review papers. Of the 42 publications identified by this search, 20 measured both peripheral insulin sensitivity and fasting EGP, 17 measured insulin sensitivity only, and five measured EGP only.

Data analysis The studies were stratified into four categories by drug (TZDs or metformin) and by study design (randomised double-blind and placebo-controlled $[\mathrm{DB} / \mathrm{PC}]$, or open-label). Since the metformin studies employed different drug doses, these data were analysed by drug dose. This distinction was not necessary for the TZD studies as these drugs were often used at the maximal dose.

The units for substrate and hormone concentrations were standardised (mmol/l for plasma glucose and $\mathrm{pmol} / \mathrm{l}$ for plasma insulin concentrations). When steady-state plasma insulin concentrations were not available, they were estimated using the regression equation of all available insulin infusion rates against the resulting steady-state insulin concentrations (regression coefficient $=12 \mathrm{pmol} / 1$ per $\left.\mathrm{mU} \min ^{-1} \mathrm{~m}^{-2}, r^{2}=0.80, p<0.001\right)$. EGP rates were all converted into micromoles per minute per kilogram. Glucose fluxes during the clamp were all converted from the original units into micromoles per minute per kilogram of fat-free mass (FFM) ( $\mu \mathrm{mol} \mathrm{min}-1 \mathrm{~kg}_{\mathrm{ffm}}{ }^{-1}$ ) by applying the following rules: (1) if FFM was not available, a fixed fraction of body weight was used $(70$ or $60 \%$ for BMIs below or above $25 \mathrm{~kg} / \mathrm{m}^{2}$, respectively); (2) data expressed per square metre of body surface area were converted into whole-body equivalents by using the given anthropometric data or, if these were not available, by using factors of 1.8 and 2.0 for lean and obese patients, respectively.

Since fasting EGP is promptly and efficiently suppressed by small increments in plasma insulin concentration according to a highly non-linear dose-response function [13], hepatic sensitivity to prevailing insulin levels was estimated by calculating a production index (PI, in $\mu \mathrm{mol}$ $\mathrm{min}^{-1} \mathrm{~kg}^{-1}$ glucose per pmol/l insulin) as the product of fasting EGP and fasting plasma insulin. Being a compounded variable, this index is rather strongly affected by the variability of the primary measurements; therefore, we only used it for between-subject comparisons. Fasting glucose clearance (in $\mathrm{ml} \mathrm{min}^{-1} \mathrm{~kg}_{\mathrm{ffm}}{ }^{-1}$ ) was calculated as the ratio of fasting glucose utilisation: fasting plasma glucose concentration.

Statistical analysis Since we did not have access to individual data, primary data were the mean values of each variable for each study group given in the original publication. These are reported in the tables as absolute values and as percentage changes from baseline following treatment. If a placebo study arm was available, the percentage changes were calculated relative to placebo. The statistics ( $p$ values) for each individual study reported in the tables are those given in the original publication.

For each variable considered, overall means were calculated for each study category (open or $\mathrm{DB} / \mathrm{PC}$ studies, metformin or TZDs) by weighting the group mean of each study by the number of patients in that study. Group means were also treated as individual data to evaluate overall means and $95 \%$ CIs by Student's $t$ test.

To compare overall means between study categories (i.e. open vs DB/PC studies, metformin vs TZD studies), the unpaired, two-tailed Student $t$ test was used to estimate the mean effect and its $95 \% \mathrm{CI}$, while the Mann-Whitney $U$-test was employed to determine the significance of the comparison. ANCOVA was used to test for group differences while controlling for potential confounders. In euglycaemic-hyperinsulinaemic clamp studies using a wide range of insulin infusion rates, insulin-mediated glucose uptake is linearly related to the logarithm of achieved steady-state plasma insulin [13]. On this basis, comparison of glucose utilisation rates at different plasma 
insulin levels across study categories was carried out by the linear fitting of glucose fluxes against the logarithmically transformed steady-state plasma insulin, and comparing the resulting regression lines by one-way ANOVA. Regression analysis was carried out by standard methods. A $p$ value of $\leq 0.05$ was considered significant.

\section{Results}

A total of 19 metformin studies and 23 TZD studies were included in the analysis. Treatment duration ranged from 2 to 26 weeks. In general, each study included a small number of patients in each treatment arm (range 6-28). After data pooling, peripheral insulin sensitivity data were available for 549 type 2 diabetic patients (metformin $n=207$, TZDs $n=342$ ) and EGP data were available for 408 patients (metformin $n=177$, TZDs $n=231$ ). Metformin use was associated with a weight loss of $0.3 \pm 1.7 \mathrm{~kg}$ (mean \pm $\mathrm{SD}$ ), whilst TZDs were associated with a weight gain of $1.8 \pm$ $1.5 \mathrm{~kg}$; the difference between the two treatments (TZDsmetformin) was $2.1 \mathrm{~kg}(95 \%$ CI 3.2-1.1).

Fasting state Both metformin and TZDs lowered fasting plasma glucose (Electronic Supplementary Material [ESM] Tables 1, 2). Metformin had a more pronounced hypoglycaemic effect than TZDs, both in open $(-28 \%$ [95\% CI -23 to -33$]$ vs $-20 \%$ [ $95 \%$ CI -14 to -24$])$ and $\mathrm{DB} / \mathrm{PC}$ studies $(-19 \%$ [95\% CI -13 to -27$]$ vs $-14 \%$ $[95 \%$ CI -9 to -19$])$. The absolute changes in plasma glucose were larger in open trials (difference $1.14 \mathrm{mmol} / 1$ [95\% CI 0.46-1.81], $p=0.003$ ) and greater with metformin than TZDs (difference $0.94 \mathrm{mmol} / \mathrm{l}$ [95\% CI $0.22-1.60$ ], $p=0.02$ ). Fasting plasma insulin concentrations decreased in most of the studies, and this change was statistically significant in the majority of the TZD studies. There was a tendency for this variable to be greater in the open vs $\mathrm{DB} /$ PC studies and with TZDs vs metformin.

Absolute EGP values ranged from $8.9-16.3 \mu \mathrm{mol} \mathrm{min}{ }^{-1}$ $\mathrm{kg}^{-1}$, and did not differ across study categories. When expressed as percentage change from baseline, EGP showed a significant decrease in the open trials, both with metformin $(-16 \%$ [95\% CI -8 to -23$])$ and TZDs $(-11 \%$ [95\% CI -3 to -18$])$. In the DB/PC studies, however, percentage EGP changes from baseline were not different from zero with either metformin or TZDs. When the effect was evaluated with respect to placebo, EGP decreased non-significantly with metformin $(-6 \%[95 \%$ CI 5 to -17$])$ and significantly with TZDs $(-12 \%$ [95\% CI -8 to -18$]$ ). When the restraining effect of insulin on EGP was accounted for by calculating the PI, it was found that the value of this variable decreased in all the individual metformin studies and in all but one of the TZD studies. Mean reductions in PI were similar for the two drugs (open studies: metformin $-30 \%$ [95\% CI -18 to -41$]$, TZDs $-33 \% \quad[95 \%$ CI -14 to -53$]$; DB/PC studies: metformin $-18 \%$ [95\% CI -4 to -33$]$, TZDs $-19 \%[95 \%$ CI -2 to -36$])$.
When plotting changes in fasting plasma glucose (FPG) against the corresponding EGP changes (Fig. 1), the majority of the datapoints fell to the right of the identity line, indicating that the reduction in FPG that occurred after administration of metformin or TZDs was larger than would be expected from the reduction in EGP alone. In support of this interpretation, an increase in fasting glucose clearance was observed in almost all studies, and the estimated effect was similar in open and DB/PC trials for both metformin (open $18 \%$ [95\% CI 5-32], DB/ PC 18\% [95\% CI 5-30]) and TZDs (open 11\% [95\% CI -1 to 22 ], DB/PC $15 \%$ [95\% CI 5-25]). In addition, the effect of metformin on both fasting plasma glucose and EGP showed a tendency to increase with dose; this was more pronounced for the latter than the former. In fact, on pooling open and DB/PC studies (which yielded similar slopes) it was estimated that fasting plasma glucose and EGP decreased by $10 \pm 3 \%(p<0.05)$ and $6 \pm 2 \%(p<0.005)$, respectively, per gram of metformin dose (Fig. 2). The effect of metformin on fasting glucose clearance was either unrelated or inversely related to dose: the parameter increased by $30 \%$ in studies involving lower doses of metformin $(<2 \mathrm{~g})$ and by $11 \%$ in studies involving $\geq 2 \mathrm{~g}$ (estimated difference in fasting glucose clearance $0.29 \mathrm{ml}$ $\mathrm{min}^{-1} \mathrm{~kg}^{-1}$ [95\% CI $\left.0.04-0.54\right]$ ).

\section{Peripheral insulin sensitivity}

In the studies where peripheral insulin sensitivity was measured, the decrease in fasting plasma glucose was similar in open (-23\% [95\% CI -19 to -26$])$ and $\mathrm{DB} / \mathrm{PC}$ $(-17 \%$ [95\% CI -14 to -23$])$ trials, and again slightly greater with metformin than TZDs $(-24 \%[95 \%$ CI -20 to -29 ] vs $-18 \%$ [95\% CI -15 to -21$], p<0.02$ ) (ESM Tables 3,4). Most metformin studies and all TZD studies measured insulin sensitivity at euglycaemia, but the exogenous insulin infusion rates employed were quite heterogeneous (range 15-600 pmol min $\mathrm{m}^{-2}$ ), yielding a wide range $(230-12,957 \mathrm{pmol} / \mathrm{l})$ of steady-state plasma insulin concentrations.

Metformin increased insulin-stimulated glucose utilisation, $M\left(R_{\mathrm{d}}\right)$, by $18 \%(95 \% \mathrm{CI} 10-26)$ and $11 \%(95 \% \mathrm{CI} 4$ 21 ) in the open and $\mathrm{DB} / \mathrm{PC}$ studies, respectively, and was reported to have reached statistical significance in $70 \%$ of the former but only $30 \%$ of the latter. In addition, when metformin's effect was evaluated against placebo, the estimated treatment-induced change did not reach statistical significance $(7 \%$ [95\% CI -8 to 22$])$. In pooled metformin studies, there was no relationship between $M\left(R_{\mathrm{d}}\right)$ changes and body weight changes $(r=0.20, p=\mathrm{ns})$.

The TZD-induced improvement in $M\left(R_{\mathrm{d}}\right)$ was statistically significant in $70 \%$ of open studies and $80 \%$ of DB/PC studies, and averaged 36\% (95\% CI 23-49) and 34\% (95\% CI 26-42) in the two study categories. Compared with placebo, TZD increased $M\left(R_{\mathrm{d}}\right)$ by $31 \%(95 \%$ CI $23-40)$. In $\mathrm{DB} / \mathrm{PC}$ studies, metformin induced a significantly $(p=0.004)$ smaller increase in $M\left(R_{\mathrm{d}}\right)$ than the TZDs 
a

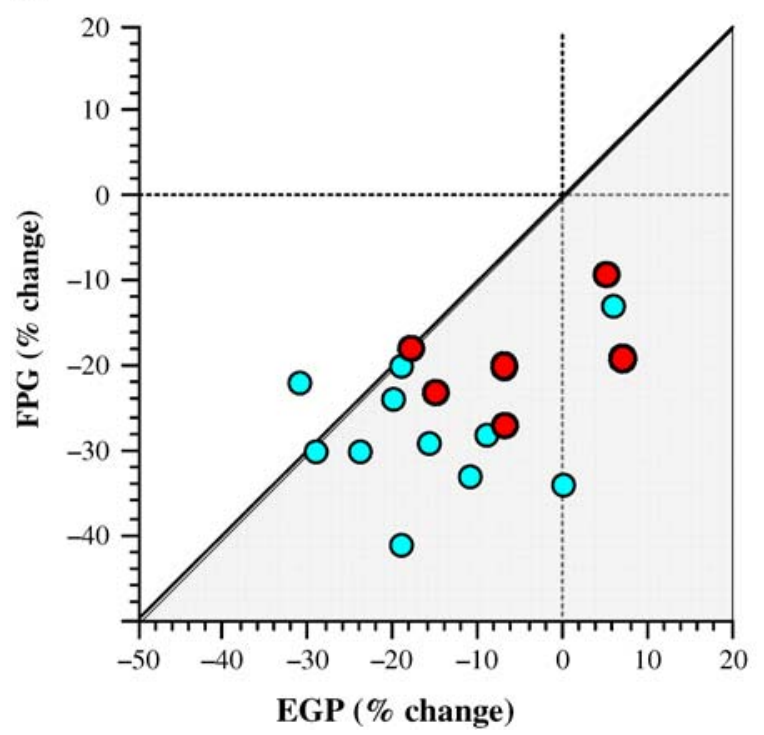

Fig. 1 Percentage change in fasting plasma glucose plotted against percentage change in EGP for open (blue circles) and placebocontrolled (red circles) studies involving administration of metformin (a) or TZDs (b). The majority of the datapoints fall to

(difference $-23 \%[95 \% \mathrm{CI}-36$ to -10$]$ or $-6.8 \mu \mathrm{mol} \mathrm{\textrm {min } ^ { - 1 }}$ $\mathrm{kg}_{\text {ffm }}{ }^{-1}$ [95\% CI -2.4 to -11.2$]$ ) (Fig. 3). In pooled TZD studies, there was no relationship between $M\left(R_{\mathrm{d}}\right)$ changes and body weight changes $(r=0.20, p=\mathrm{ns})$.

To confirm these findings, $M\left(R_{\mathrm{d}}\right)$ was regressed against the logarithmically transformed steady-state plasma insulin concentrations (SSPI) for pooled open and DB/PC studies (i.e. construction of a dose-response curve). Pretreatment fitting functions were similar $(p=0.35)$ for metformin $(y=$ $\left.-32+18 \log _{10}[\mathrm{SSPI}], r=0.69, p=0.0002\right)$ and TZDs $(y=-32$

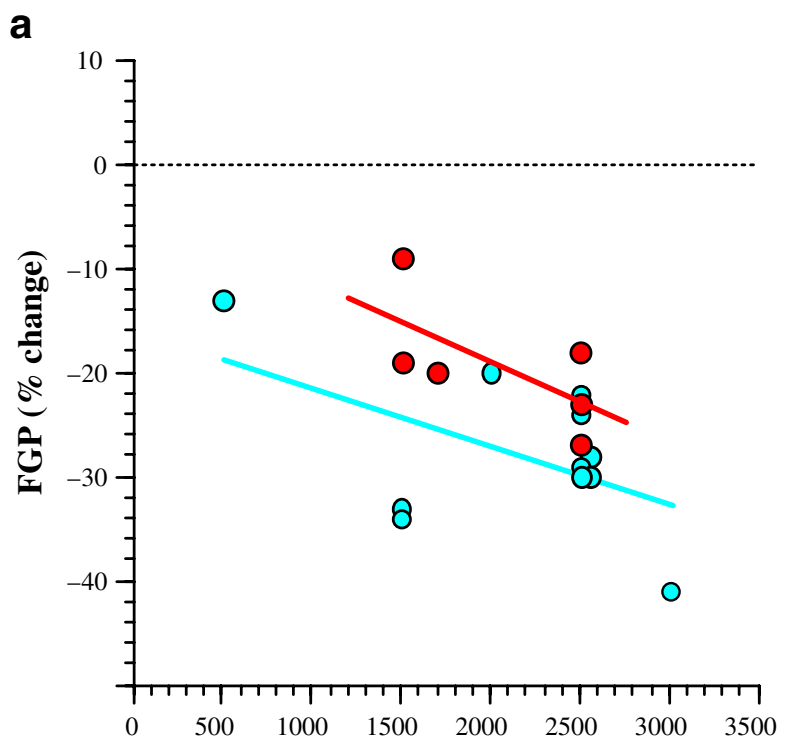

Metformin dose (mg/day) b

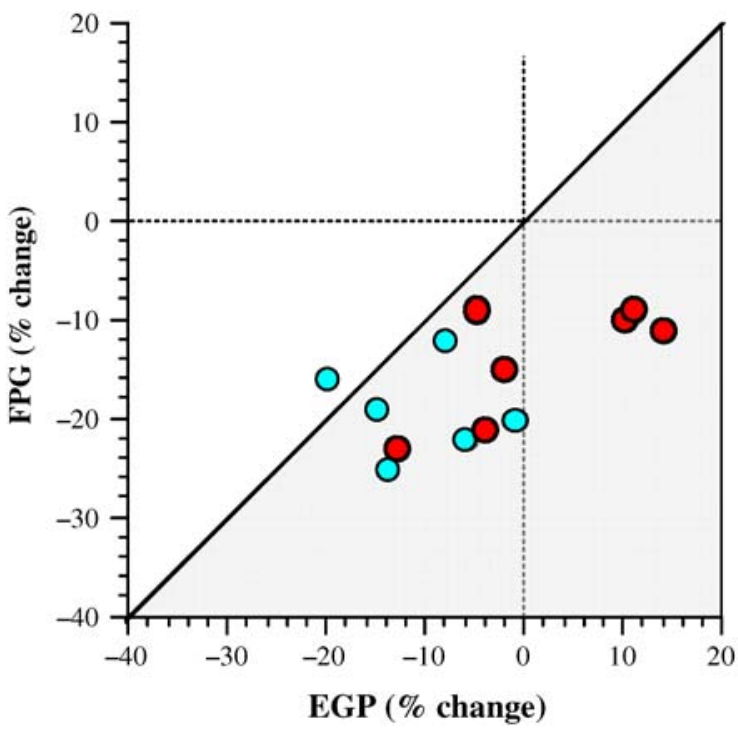

the right of the line of identity, indicating that the reduction in FPG that occurred after treatment was larger than would be expected from the reduction in EGP alone

$\left.+19 \log _{10}[\mathrm{SSPI}], r=0.65, p<0.0001\right)$. After treatment, the regression lines for metformin $\left(y=-42+23 \log _{10}[\mathrm{SSPI}]\right.$, $r=0.75, p<0.0001)$ and TZDs $\left(y=-51+28 \log _{10}[\mathrm{SSPI}\right.$, $r=0.79, p<0.0001)$ were significantly $(p=0.005)$ different from each other (Fig. 4). When glucose fluxes were corrected for the prevailing glucose concentration by calculating glucose clearances, the estimated effects of both metformin and TZDs, as well as the comparison between the two (as percentage changes) were superimposable with those reported above for $M\left(R_{\mathrm{d}}\right)$.

b

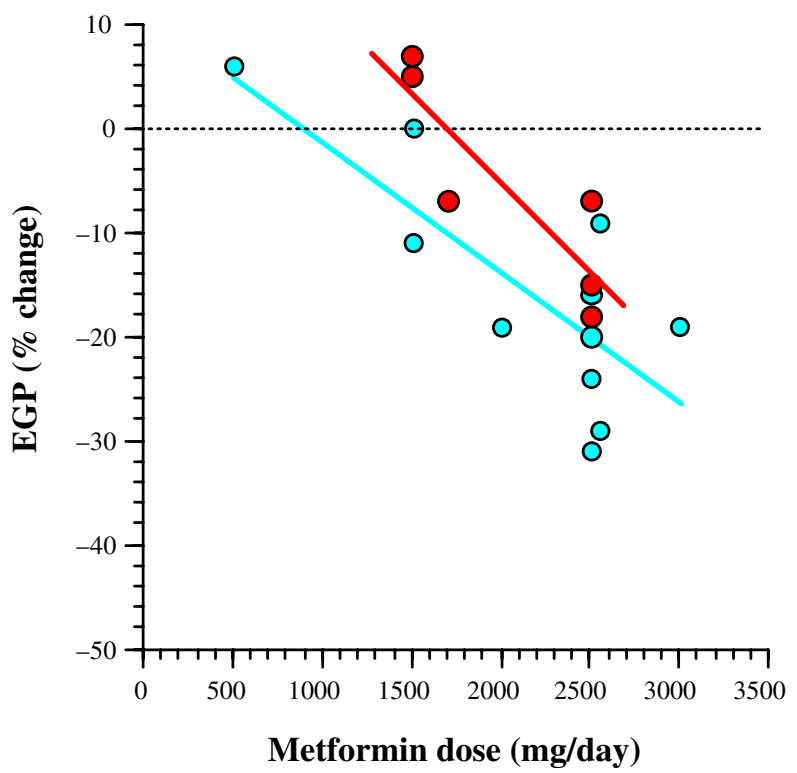

Fig. 2 The relationships between daily dose of metformin and percentage change in fasting plasma glucose (a) and EGP (b) in open (blue circles, blue lines) and placebo-controlled studies (red circles, red lines) 


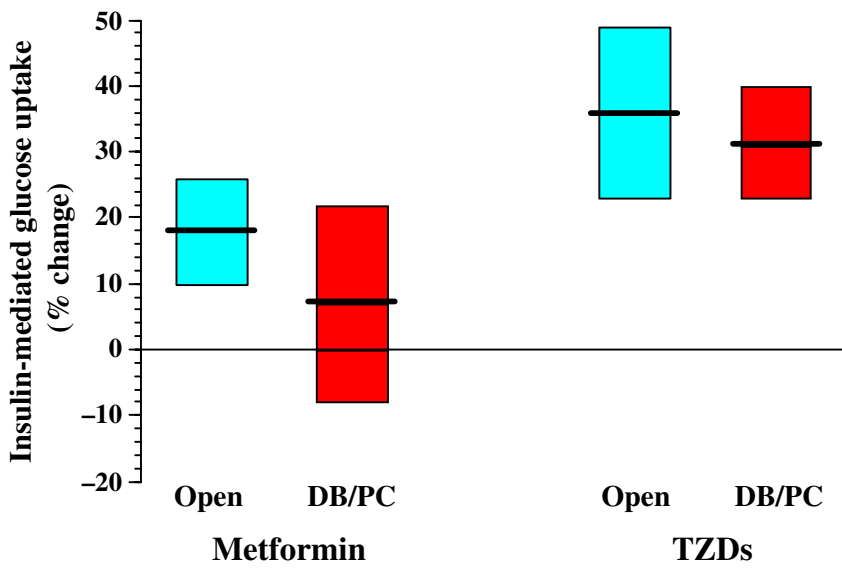

Fig. 3 Treatment-induced changes in insulin-mediated glucose uptake ( $M$ value) with metformin and TZDs. Bars are means and $95 \%$ CIs. Open, open clamp studies (change from baseline); $\mathrm{DB} / \mathrm{PC}$, double-blind, placebo-controlled clamp studies (placebo-adjusted change)

\section{Discussion}

Our analysis shows that PPAR $\gamma$ agonists and metformin reduce plasma glucose levels by partially different mechanisms. Metformin dose-dependently depresses EGP and increases fasting glucose clearance, but does not have a significant effect on insulin-mediated glucose disposal. PPAR $\gamma$ agonists, on the other hand, clearly potentiate insulin-stimulated glucose disposal over a wide range of insulin concentrations, whilst also improving EGP insulin sensitivity and fasting glucose clearance. This general result requires more detailed consideration.

First, the patient groups in the different studies had a fairly common phenotype: middle-aged, overweight subjects (data not shown) with an average fasting plasma glucose of $\sim 11 \mathrm{mmol} / \mathrm{l}$ and a range of $8.2-15.9 \mathrm{mmol} / \mathrm{l}$ (ESM Table 1). In general, the absolute hypoglycaemic effect of a glucose-lowering agent is proportional to the magnitude of pretreatment FPG. When expressed as a percentage of baseline, the decrease in FPG elicited by a particular agent thus tends to remain constant. We therefore minimised the effects of different initial severities of hyperglycaemia by expressing treatment-induced changes in FPG in this way. Second, the effects of treatment were qualitatively similar in both open and $\mathrm{DB} / \mathrm{PC}$ studies, but were generally larger in open studies. This necessitated separate analyses of the two study types. Third, after controlling for these sources of variability, the effect of
Fig. 4 Data from open and placebo-controlled studies were pooled to examine the relationship between insulin-mediated glucose uptake ( $M$ value) and steady-state plasma insulin concentrations (logarithmic scale) before (pink circles/squares) and after (dark blue circles/squares) administration of metformin (a) or TZDs (b). The graphs show the results of regression analysis (solid lines) and associated $95 \%$ CIs (dotted lines). The shaded area encompasses the physiological range of postprandial insulin concentrations

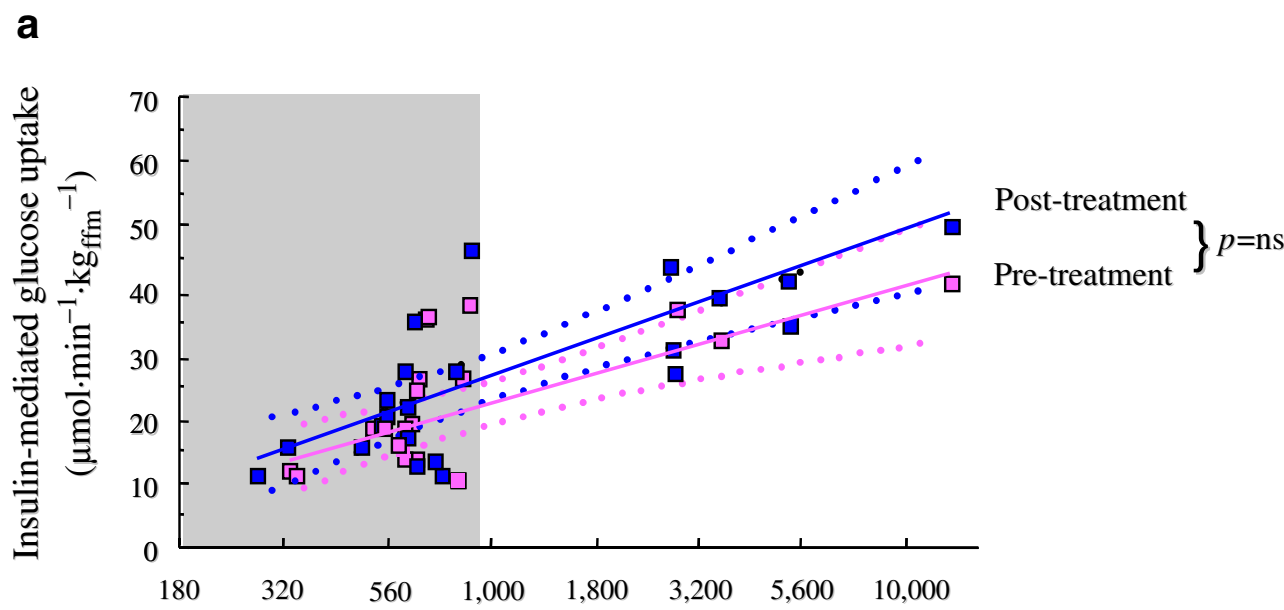

b

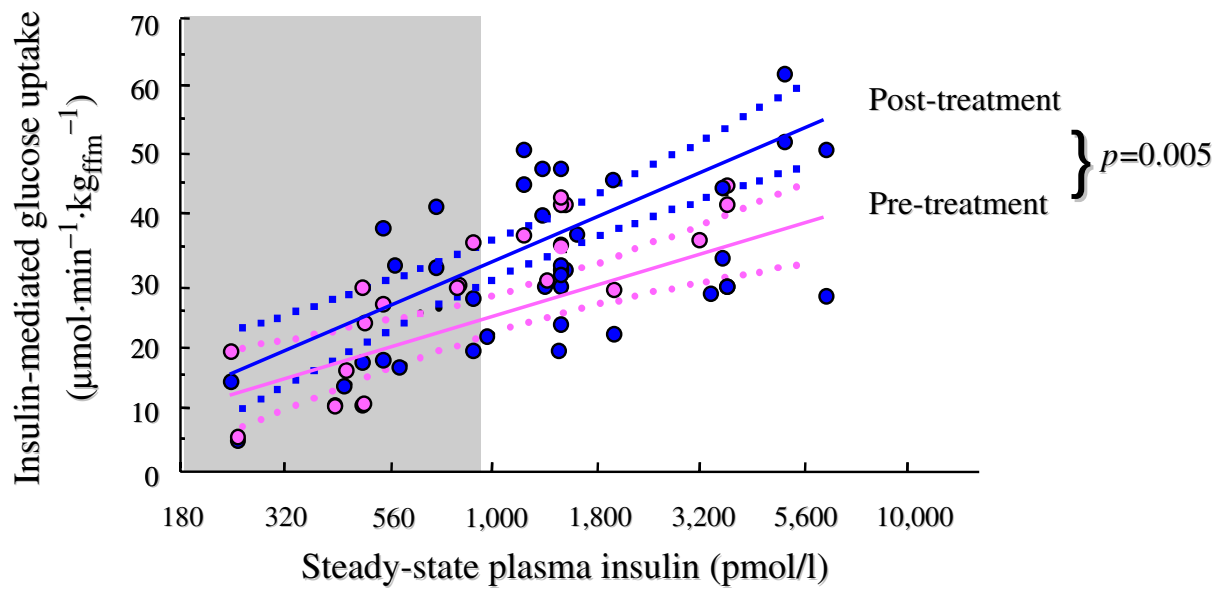


metformin on fasting plasma glucose was slightly $(0.94 \mathrm{mmol} / \mathrm{l})$, but significantly, greater than that of TZDs. The difference remained statistically significant after controlling for changes in body weight. One explanation for this finding may be the differences in the dose ranges examined: although both agents were administered at concentrations across their approved range, this is wider for metformin $(0.5-3.0 \mathrm{~g} /$ day $)$ than for TZDs (except troglitazone). Nonetheless, it is important to emphasise that none of the studies reviewed here was designed to assess differences in treatment efficacy. Comparative clinical trials $[14,15]$ have shown similar $\mathrm{HbA}_{1 \mathrm{c}}$ reductions with metformin and TZDs, particularly in the longer term ( $>20$ weeks).

Fourth, even when using percentage changes from baseline, the effect of metformin on fasting EGP was inconsistent in placebo-controlled studies. This rather unexpected finding may be due to variations in the experimental protocol employed (duration of fast, type of tracer, equilibration time). Also, the effect of TZDs on fasting EGP, if statistically significant, was rather small $(-11 \%$ on average). On the other hand, when EGP was 'adjusted' for the prevailing plasma insulin concentrations in the present analysis (by calculating the PI), the results with metformin and TZDs converged: both drugs decreased PI by $\sim 20 \%$ compared with placebo. Clearly, the drop in fasting plasma insulin (and also in plasma glucose) that both treatments induce explains why absolute rates of EGP may not be significantly changed by treatment, despite improved hepatic insulin sensitivity. Independent support for a direct hepatic effect of TZDs comes from an elegant study [16] showing that, in type 2 diabetic patients, rosiglitazone reduced EGP early during the course of treatment, before there were any detectable changes in fasting plasma glucose or fasting plasma NEFA.

With either drug treatment, the magnitude of change in fasting plasma glucose was greater than could be accounted for by the corresponding decline in fasting EGP (Fig. 1). The other determinant of plasma glucose concentration in the postabsorptive state is glucose clearance, which was significantly enhanced by both metformin and TZDs. The mechanism underlying this effect is unclear. In the fasting state, it is estimated that $\sim 70 \%$ of glucose uptake occurs in non-insulin-sensitive tissues (mostly brain and erythrocytes) [13]. Insulin target tissues (skeletal muscle, fat and liver) account for the remainder of fasting glucose disposal. It is possible that either treatment improves both pathways by attenuating the deleterious effect of chronic hyperglycaemia (glucose toxicity) and/or through potentiatiation of glucose uptake by insulin in target tissues by the same mechanisms as those that occur in the fully insulinised state (particularly fat tissue in the case of TZDs). Interestingly, this pharmacological effect is rarely (for an example, see [17]) mentioned in discussions of mode of drug action, despite the fact that its relative contribution to lowering fasting plasma glucose is almost equivalent to that of improved suppression of EGP.

With regard to treatment-induced changes in peripheral insulin sensitivity, our analysis indicates that TZDs are insulin-sensitising agents (Fig. 3). In contrast, metformin does not improve peripheral insulin sensitivity, particularly over the range of physiological plasma insulin concentrations (Fig. 4). Of note, pretreatment $M\left(R_{\mathrm{d}}\right)$ values (which ranged from 5-60 $\mu \mathrm{mol} \mathrm{min}{ }^{-1} \mathrm{~kg}_{\mathrm{ffm}}{ }^{-1}$ across all studies) were similar in metformin and TZD studies (Fig. 4). At a plasma insulin concentration of $\sim 600 \mathrm{pmol} / 1$ (corresponding to an exogenous insulin infusion rate of $40 \mathrm{mU} \mathrm{min}^{-1}$ $\left.\mathrm{m}^{-2}\right)$, the $M\left(R_{\mathrm{d}}\right)$ value predicted from the regression (including all baseline studies) was $20 \mu \mathrm{mol} \mathrm{min}{ }^{-1}$ $\mathrm{kg}_{\mathrm{ffm}}{ }^{-1}$. This represents a reduction of at least $50 \%$ in $M\left(R_{\mathrm{d}}\right)$ compared with the mean value measured in 350 nondiabetic subjects of comparable age (59 years) and obesity (BMI $28 \mathrm{~kg} / \mathrm{m}^{2}$ ) clamped at the same plasma insulin concentrations (European Group for the study of Insulin Resistance [EGIR] database [18]).

The insulin-sensitising effect of TZDs was small in absolute value (mean $7 \mu \mathrm{mol} \mathrm{min}{ }^{-1} \mathrm{~kg}_{\mathrm{ffm}}{ }^{-1}$ across insulin doses) but substantial in relative terms $(\sim 30 \%)$ for an insulin-resistant population of type 2 patients. The corresponding effect of metformin was smaller and more variable, and hence not statistically significant (Figs. 3, 4). The controversy surrounding the insulin sensitising action of metformin on peripheral tissues may be explained by glucose toxicity. It is known that chronic hyperglycaemia causes deterioration of both beta cell function and insulin action $[19,20]$, and that these effects are reversed by improved glycaemic control $[21,22]$. As a result, measurable improvements in insulin secretion and action may occur after administration of any antihyperglycaemic treatment, purely as a result of improved glycaemic control. It can therefore be difficult to separate the direct effects of drug treatment from this attenuation of glucotoxicity. In a placebo-controlled study in type 2 diabetic patients [23], metformin and rosiglitazone were dosed so as to achieve equivalent glycaemic control at the end of 16 weeks of treatment. At this time, clamp-derived insulin sensitivity was significantly improved with rosiglitazone and unchanged with metformin. Likewise, in a comparative study of troglitazone $(600 \mathrm{mg} /$ day $)$ and metformin (1.7 g/day) [24], diabetic patients were rendered euglycaemic (by continuous subcutaneous insulin infusion) for 4 weeks prior to, and during, drug treatment. Under these conditions, insulin-stimulated peripheral glucose disposal was increased by troglitazone but not by metformin. Further support for this conclusion is provided by a recent placebo-controlled comparison of the effects of metformin and rosiglitazone in newly diagnosed type 2 diabetes patients. In this study, insulin-mediated glucose uptake was enhanced by rosiglitazone but not by metformin. This improvement occurred both at the whole-body level and in the leg (a predominantly muscular region), and 
was not associated with the typical changes in intracellular insulin signalling and gene expression that occur when glucose toxicity is alleviated [25]. It should be noted, however, that some studies have reached completely opposite conclusions. For example, Hother-Nielsen et al. [26] — a study that is included in this review - concluded that metformin has no hepatic effect and that the improved glycaemic control associated with its use is due to increased peripheral glucose utilisation. These contradictory results emphasise the importance of considering all pertinent data, as has been done in the present review.

Studies in which TZDs were administered to nondiabetic, insulin-resistant subjects - including lean, obese and hypertensive individuals, and first-degree relatives of patients with type 2 diabetes [27-31] —were not included in the present analysis. With only one exception [27], however, they also showed a consistent reduction in PI and improvements in peripheral insulin sensitivity.

The limitation of this study is that a proper meta-analysis could not be carried out because of the difficulty of retrieving individual data from 42 different studies, some of which date back almost two decades. However, by converting relevant parameters into common units and by performing a statistical analysis of the original mean values, consistent, robust information was obtained. Thus, the quantitative relationship between $M\left(R_{\mathrm{d}}\right)$ and steadystate plasma insulin concentrations was surprisingly consistent across many different studies (Fig. 4). From this dose-response curve, it can be estimated that insulinmediated glucose utilization at typical postprandial insulin

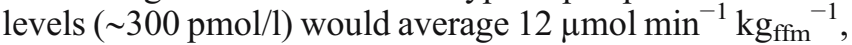
and that doubling this rate, i.e. restoring it to normal levels [13], would require a ten-fold increase in plasma insulin concentrations. This calculation underscores the notion that sensitising tissues to insulin action in type 2 diabetes is certainly useful but hardly sufficient to restore glucose tolerance.

Duality of interest E. Ferrannini has received educational sponsorship from AstraZeneca. Editorial support was provided by The Future Forum Secretariat, London, UK. The Future Forum is sponsored by AstraZeneca.

\section{References}

1. Henry RR (2003) Insulin resistance: from predisposing factor to therapeutic target in type 2 diabetes. Clin Ther 25(Suppl B): B47-B63

2. Olefsky JM (2000) Treatment of insulin resistance with peroxisome proliferator-activated receptor gamma agonists. J Clin Invest 106:467-472

3. Groop LC, Widen E, Ferrannini E (1993) Insulin resistance and insulin deficiency in the pathogenesis of type 2 diabetes (noninsulin-dependent) diabetes mellitus: errors of metabolism or of methods. Diabetologia 36:1326-1331

4. Johnson AB, Webster JM, Sum CF et al (1993) The impact of metformin therapy on hepatic glucose production and skeletal muscle glycogen synthase activity in overweight type II diabetic patients. Metabolism 42:1217-1222
5. Perriello G, Misericordia P, Volpi E et al (1994) Acute antihyperglycemic mechanisms of metformin in NIDDM. Evidence for suppression of lipid oxidation and hepatic glucose production. Diabetes 43:920-928

6. Inzucchi SE, Maggs DG, Spollett GR et al (1998) Efficacy and metabolic effects of metformin and troglitazone in type II diabetes mellitus. N Engl J Med 338:867-872

7. Hundal HS, Ramlal T, Reyes R, Leiter LA, Klip A (1992) Cellular mechanism of metformin action involves glucose transporter translocation from an intracellular pool to the plasma membrane in L6 muscle cells. Endocrinology 131:1165-1173

8. Sirtori CR, Pasik C (1994) Re-evaluation of a biguanide, metformin: mechanism of action and tolerability. Pharmacol Res 30:187-228

9. Hällsten K, Virtanen KA, Lonnqvist F et al (2002) Rosiglitazone but not metformin enhances insulin- and exercisestimulated skeletal muscle glucose uptake in patients with newly diagnosed type 2 diabetes. Diabetes 51:3479-3485

10. Hundal RS, Inzucchi SE (2003) Metformin: new understandings, new uses. Drugs 63:1879-1894

11. Ikeda T, Iwata K, Murakami H (2000) Inhibitory effect of metformin on intestinal glucose absorption in the perfused rat intestine. Biochem Pharmacol 59:887-890

12. Cuber JC, Bosshard A, Vidal H, Vega F, Wiernsperger N, Rapin JR (1994) Metabolic and drug distribution studies do not support direct inhibitory effects of metformin on intestinal glucose absorption. Diabetes Metab 20:532-539

13. Ferrannini E, DeFronzo RA (2004) Insulin actions in vivo: glucose metabolism. In: DeFronzo RA, Ferrannini E, Keek H, Zimmet P (eds) International textbook of diabetes mellitus, 3rd edition. John Wiley and Sons, Chichester, pp 277-302

14. Pavo I, Jermedy J, Varkonyi TT et al (2003) Effect of pioglitazone compared with metformin on glycemic control and indicators of insulin sensitivity in recently diagnosed patients with type 2 diabetes. J Clin Endocrinol Metab 88:1637-1645

15. Schernthaner G, Matthews DR, Charbonnel B, Hanefeld M, Brunetti P; Quartet Study Group (2005) Efficacy and safety of pioglitazone versus metformin in patients with type 2 diabetes mellitus: a double blind randomized trial. J Clin Endocrinol Metab 89:6068-6076

16. Tonelli J, Li W, Kishore P et al (2004) Mechanisms of early insulin-sensitizing effects of thiazolidinediones in type 2 diabetes. Diabetes 53:1621-1629

17. McIntyre HD, Ma A, Bird DM, Paterson CA, Ravenscroft PJ, Cameron DP (1991) Metformin increases insulin sensitivity and basal glucose clearance in type 2 (non-insulin dependent) diabetes mellitus. Aust N Z J Med 21:714-719

18. Ferrannini E, Natali A, Bell P, Cavallo-Perin P, Lalic N, Mingrone $G$ (1997) Insulin resistance and hypersecretion in obesity. European Group for the Study of Insulin Resistance (EGIR). J Clin Invest 100:1166-1173

19. Robertson RP, Zhang HJ, Pyzdrowski KL, Walseth TF (1992) Preservation of insulin mRNA levels and insulin secretion in HIT cells by avoidance of chronic exposure to high glucose concentrations. J Clin Invest 90:320-325

20. Boden G, Ruiz J, Kim CJ, Chen X (1996) Effects of prolonged glucose infusion on insulin secretion, clearance, and action in normal subjects. Am J Physiol 270:E251-E258

21. Andrews WJ, Vasquez B, Nagulesparan M et al (1984) Insulin therapy in obese, non-insulin-dependent diabetes induces improvements in insulin action and secretion that are maintained for two weeks after insulin withdrawal. Diabetes 33:634-642

22. Garvey WT, Olefsky JM, Griffin J, Hamman RF, Kolterman OG (1985) The effect of insulin treatment on insulin secretion and insulin action in type II diabetes mellitus. Diabetes 34: $222-234$ 
23. Natali A, Baldeweg S, Toschi E et al (2004) Vascular effects of improving metabolic control with metformin or rosiglitazone in type 2 diabetes. Diabetes Care 27:1349-1357

24. Yu JG, Kruszynska YT, Mulford MI, Olefsky JM (1999) A comparison of troglitazone and metformin on insulin requirements in euglycemic intensively insulin-treated type 2 diabetic patients. Diabetes 48:2414-2421

25. Karlsson HK, Hallsten K, Bjornholm M et al (2005) Effects of metformin and rosiglitazone treatment on insulin signaling and glucose uptake in patients with newly diagnosed type 2 diabetes: a randomized controlled study. Diabetes 54:1459-1467

26. Hother-Nielsen O, Schmitz O, Andersen PH, Beck-Nielsen H, Pedersen O (1989) Metformin improves peripheral but not hepatic insulin action in obese patients with type II diabetes. Acta Endocrinol (Copenh) 120:257-265

27. Kruszynska YT, Yu JG, Olefsky JM, Sobel BE (2000) Effects of troglitazone on blood concentrations of plasminogen activator inhibitor 1 in patients with type 2 diabetes and in lean and obese normal subjects. Diabetes 49:633-639
28. Raji A, Seely EW, Bekins SA, Williams GH, Simonson DC (2003) Rosiglitazone improves insulin sensitivity and lowers blood pressure in hypertensive patients. Diabetes Care 26: 172-178

29. Nolan JJ, Ludvik B, Beerdsen P, Joyce M, Olefsky J (1994) Improvement in glucose tolerance and insulin resistance in obese subjects treated with troglitazone. $\mathrm{N}$ Engl J Med 331:1188-1193

30. Meyer MM, Levin K, Grimmsmann T et al (2002) Troglitazone treatment increases protein kinase B phosphorylation in skeletal muscle of normoglycemic subjects at risk for the development of type 2 diabetes. Diabetes 51:2691-2697

31. Levin K, Hother-Nielsen O, Henriksen JE, Beck-Nielsen $\mathrm{H}$ (2004) Effects of troglitazone in young first-degree relatives of patients with type 2 diabetes. Diabetes Care 27:148-154 